\title{
Diet/lifestyle and risk of diabetes and glycemic traits: a Mendelian randomization study
}

\author{
Renyu Ding ${ }^{1}$, Tao Huang ${ }^{2^{*}}$ and Jiali Han ${ }^{3^{*}}$
}

\begin{abstract}
Background: Observational studies have demonstrated diet/lifestyle play roles in development of type 2 diabetes (T2DM); however, it remains unclear whether these relationships are causal.

Methods: A two-sample MR approach was used to examine the causal effect of diet/lifestyle upon risk of T2DM and glycemic traits.

Results: The protein intake-increasing allele $\mathrm{C}$ of FTO was significant associated with higher risk of T2DM (Beta \pm SE $=$ $0.104 \pm 0.014, P=4.40 \times 10^{-11}$ ), higher level of HOMA-IR (Beta $\pm S E=0.016 \pm 0.004, P=9.55 \times 10^{-5}$ ), HOMA-B (Beta $\pm \mathrm{SE}=0.008 \pm 0.003, P=0.020$ ). Using MR analyses, increased protein intake was causally associated with an increased risk of T2DM (Beta $\pm S E=0.806 \pm 0.260, P=0.002$ ). In addition, smoking cessation was causally associated with increased levels of glycemic traits such as HOMA-IR (Beta $\pm S E=0.165 \pm 0.072, P=0.021$ ), fasting insulin (Beta $\pm \mathrm{SE}=0.132 \pm 0.066, P=0.047)$ and fasting glucose (Beta $\pm \mathrm{SE}=0.132 \pm 0.064, P=0.039$ ).
\end{abstract}

Conclusions: These results provide evidence supporting a causal role for higher protein intake and smoking cession in T2DM. Our study provides further rationale for individuals at risk for diabetes to keep healthy lifestyle.

\section{Background}

Diabetes has become a worldwide epidemic, with an estimated more than 387 million people with diabetes in 2014. However, the etiology of diabetes is poorly understood. Identifying potentially causal risk factors could help guide prevention efforts.

Compelling evidence showed that excessive caloric intake is a major driving force behind escalating obesity and type 2 diabetes (T2DM) epidemics, Importantly, diet quality of fats, carbohydrates and protein, [1] and unhealthy lifestyles have been also implicated in the rapid rise of T2DM [1]. For example, cigarette smoking is a well-established risk factor for T2DM, and smoking cessation leads to higher short-term risk [2]. In addition, both short and long sleep duration are associated with a significantly increased risk of T2DM [3]. However, it

\footnotetext{
* Correspondence: huangtao@bjmu.edu.cn; jiali_han@163.com

${ }^{2}$ Department of Epidemiology and Biostatistics, School of Public Health,

Peking University, Beijing 100191, China

${ }^{3}$ Department of Otolaryngology, The First Hospital of China Medical

University, Liaoning 110001, China

Full list of author information is available at the end of the article
}

remains uncertain whether these relationshipsare causal since it is difficult to fully protect observational studies from bias due to reverse causation or confounding.

In the absence of high-quality RCT data, the principles of Mendelian randomization (MR) can be applied to strengthen or refute the causality of diet/lifestyle in T2DM etiology [4]. This approachis based on the principle that genetic variants are randomly allocated at meiosis, and consequently are independent of many factors that bias observational studies, such as confounding and reverse causation [4]. Therefore, MR is conceptually similar to an RCT.

Recent advances in GWAS have substantially improved our understanding of genetic roles in diet/lifestyle such as macronutrients, $[5,6]$ cigarette smoking, [7] smoking initiation, [7] smoking cessation, [7] sleep, [8] allowing us to use MR to estimate their causal effects. Therefore, we undertook an MR analysis using summary statistics from DIAGRAM for T2DM and GAGIC consortium for glycemic traits, respectively to estimate the causal effects of diet/lifestyle factors on risk of T2DM and glycemic traits. 


\section{Methods}

\section{SNP selection and data sources}

The design of our study had three key components: 1) the identification of genetic variants to serve as instrumental variable (IV) for diet/lifestyle; 2) the acquisition of summary datafor the genetic variants from the DIAGRAM and MAGIC consortium; and 3) the estimate of causal effect of diet/lifestyleon T2DM and glycemic traits.

We searched the GWAS catalog to identify single nucleotide polymorphisms (SNPs) associated with diet/lifestyle [9]. We included all SNPs identified from original study reports for diet/lifestyle such as macronutrients, [5, 6] cigarette smoking, [7] smoking initiation, [7] smoking cessation, [7] sleep [8] in the GWAS catalog as potential IV. Effect estimates of these diet/lifestyle-associated SNPs on the risk of T2DM [10] and glycemic traits such as HOMA-IR, [11] HOMA-B, [11] fasting insulin, [11] fasting glucose, [11] Hba1c, [12] 2hGLU [13] were assessed using the summary statistics from the DIAGRAM consortium and MAGIC consortium, respectively. Summary statistics from these consortia can be downloaded at the following links: DIAGRAM consortium, http://diagram-consortium.org/; MAGIC consortium, https://www.magicinvestigators.org/.Cohorts participating in the DIAGRAM consortium and MAGIC consortium received ethics approval from local institutional review boards and informed consent from all participants.

We harmonized the data from the summary statistics of consortium [14]. First, we identified variants that do not share the same allele pair between datasets, and then correct this if possible. Second, we identified variants with unmatched effect and other alleles and then 'flip' their effect estimates and effect allele frequencies in only one of the datasets.

\section{MR estimates and statistical analyses}

Inference of causality in the estimated etiological associations between diet/lifestyle and risk of T2DM and glycemic traits is based on three MR assumptions [15]. The selected genetic variants are valid instrumental variables if these three assumptions are satisfied (Fig. 1). Three assumptions are a) the genetic variant (instrumental variable) is associated with exposure (diet/lifestyle); b) the genetic variant is not associated with confounders; and c) the genetic variant is associated with outcomes (T2DM and glycemic traits) only through their effect on exposure, not through other pathways.

Here, we employed the previously described methods to examine the causal association of diet/lifestyle with T2DM and glycemic traits [16]. We obtained the effect estimates of the selected SNPs on diet/lifestyle, and corresponding effect estimates for the selected SNPs on
T2DM and glycemic traits were extracted from the DIAGRAM and MAGIC consortium. A two-sample MR approach were applied to examine causal effect. This two-sample approach has equivalent statistical power to one-sample approaches [17] and is favorable in this setting since large GWAS consortium and are thus better powered than an MR study in a single cohort with a smaller sample size. This approach weighted the effect estimate of each SNP on T2DM and glycemic traits by its effect on diet/lifestyle. These estimates were then pooled using a fixed meta-analytic model to produce a summary measure of the effect of diet/lifestyle on T2DM and glycemic traits [18].

$P$ values were two-sided and evidence of association was declared at $P<0.05$. Where indicated, Bonferroni corrections were used to make allowance for multiple testing, although this is likely to be overly conservative given the non-independence of many of the outcomes tested. All analyses were performed in $\mathrm{R}$ version 3.1.2 and Stata release 13.1 (StataCorp, College Station, TX).

\section{Results \\ SNP selection}

Overall, we identified $2,2,1,8,3,1$, 1that achieved genome-wide significance for carbohydrate intake, protein intake, fat intake, smoking, smoking initiation, smoking cessation, respectively. The SNPs for carbohydrate intake, protein intake, smoking, smoking initiation, and sleep duration are LD-independent SNPs. Therefore, in total we used 15 SNPs for our MR analysis, as shown in Table 1.

\section{Association of the selected SNPs with diet/lifestyle}

Table 1 displays the SNPs that identified in GWAS for diet/lifestyle and describes their associations with carbohydrate intake, [6] protein intake, [5, 6] fat intake, [6] smoking (number), [7] smoking initiation, [7] smoking cessation, [7] and sleep [8]. Each of these SNPs explained an important proportion of the population-level variance in diet/lifestyle.

\section{Association of the selected SNPs with T2DM and glycemic traits}

We found that protein intake SNP rs1421085 at FTO was significantly associated risk of T2DM and glycemic traits (Table 2). The protein intake-increasing allele $\mathrm{C}$ of FTO was significant associated with higher risk of $\mathrm{T} 2 \mathrm{DM}$ (Beta $\pm \mathrm{SE}=0.104 \pm 0.014, \quad P=4.40 \times 10^{-11}$ ), higher level of HOMA-IR (Beta $\pm \mathrm{SE}=0.016 \pm 0.004, P=$ $\left.9.55 \times 10^{-5}\right)$, HOMA-B (Beta $\pm \mathrm{SE}=0.008 \pm 0.003, P=$ 0.020), Hba1c (Beta $\pm \mathrm{SE}=0.008 \pm 0.004, P=0.028$ ), whereas lower level of fasting insulin (Beta $\pm \mathrm{SE}=-0.015$ $\left.\pm 0.004, P=7.48 \times 10^{-5}\right)$. In addition, the $G$ allele of SNP rs3025343 at DBH forsmoking cessation was significantly 


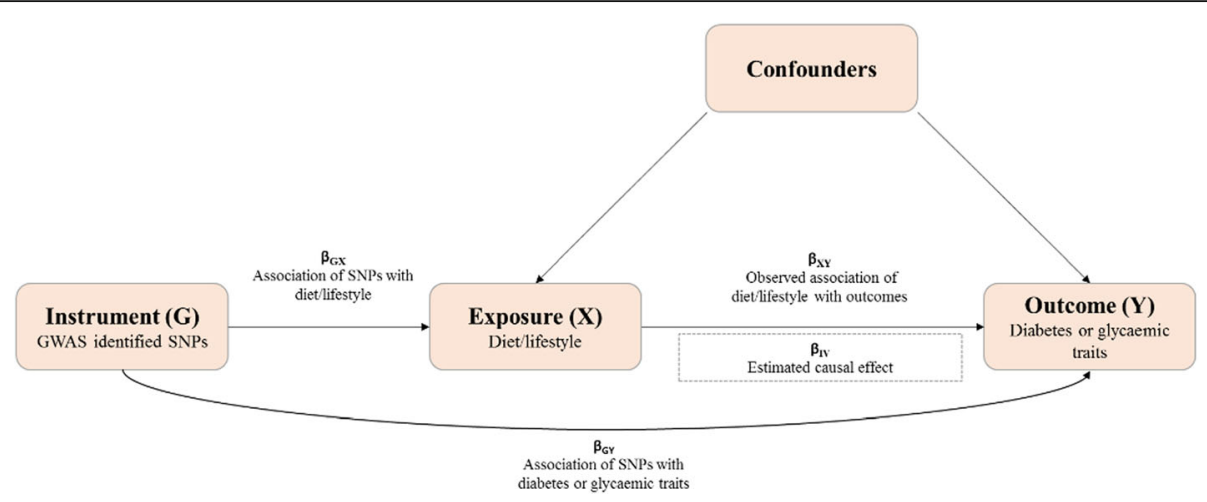

Fig. 1 Schematic representation of an MR analysis. This diagram shows that SNPs associated with diet/lifestyle were selected from the GWAS studies. Corresponding effect estimates for these SNPs upon diabetes and glycemic traits were obtained from the DIAGRAM and MAGIC consortium. Because of the randomization of alleles at meiosis, SNPs are not associated with confounding variables that may bias estimates obtained from observational studies

associated with higher level of HOMA-IR (Beta $\pm \mathrm{SE}=$ $0.020 \pm 0.008, P=0.010$ ), fasting insulin (Beta $\pm \mathrm{SE}=$ $0.016 \pm 0.008, P=0.032$ ), and fasting glucose (Beta \pm $\mathrm{SE}=0.016 \pm 0.007, P=0.029$ )

\section{MR estimates}

Using MR analyses, we found that increased percentages of total energy consumption from protein was causally associated with an increased risk of T2DM (Beta $\pm \mathrm{SE}=$ $0.806 \pm 0.260, P=0.002$ ) (Table 3 ). TheI ${ }^{2}$ estimate for heterogeneity was $0 \%(95 \%$ CI $0 \%-12 \%)$. However, increased percentages of total energy consumption from protein was not associated with glycemic traits (Table 3 ). In addition, our results also suggested that smoking cessation was causally associated with increased levels of glycemic traits such as HOMA-IR (Beta $\pm \mathrm{SE}=$ $0.165 \pm 0.072, P=0.021$ ), fasting insulin (Beta $\pm \mathrm{SE}=$ $0.132 \pm 0.066, P=0.047$ ) and fasting glucose (Beta \pm $\mathrm{SE}=0.132 \pm 0.064, P=0.039$ ). However, we did no observe significant association between fat intake, carbohydrate intake, sleep duration, and smoking initiation and number of cigarettes and risk of T2DM and glycemic traits (Table 3).

\section{Discussion}

Using a MR study design, we found that genetically elevated protein intake and smoking cession are causally associated with an increased risk of T2DM and higher

Table 1 Characteristics of single nucleotide polymorphisms used as instrumental variables

\begin{tabular}{|c|c|c|c|c|c|c|c|c|}
\hline \multirow[t]{2}{*}{ Lifestyle factors } & \multirow{2}{*}{$\begin{array}{l}\text { Lifestyle-Associated } \\
\text { SNP }\end{array}$} & \multirow{2}{*}{$\begin{array}{l}\text { Nearest } \\
\text { Gene(s) }\end{array}$} & \multirow[t]{2}{*}{ Chr } & \multirow{2}{*}{$\begin{array}{l}\text { effect } \\
\text { allele }\end{array}$} & \multirow{2}{*}{$\begin{array}{l}\text { Effect allele } \\
\text { frequency }\end{array}$} & \multicolumn{3}{|c|}{ lifestyle } \\
\hline & & & & & & Beta & SE & $P$ \\
\hline Carbohydrate [5] & rs10163409 & FTO & 16 & $A$ & 0.69 & 0.19 & 0.05 & $2.2 \times 10^{-4}$ \\
\hline Carbohydrate [5] & rs197273 & TANK & 2 & A & 0.48 & 0.23 & 0.04 & $9.6 \times 10^{-8}$ \\
\hline Carbohydrate [6] & rs838145 & IZUMO1 & 19 & G & 0.46 & 0.25 & 0.04 & $1.68 \times 10^{-8}$ \\
\hline Protein [6] & rs1421085 & FTO & 16 & C & 0.42 & 0.09 & 0.023 & $4.80 \times 10^{-7}$ \\
\hline Protein [5] & rs838133 & FGF21 & 19 & G & 0.55 & 0.11 & 0.02 & $7.9 \times 10^{-9}$ \\
\hline Fat [6] & rs838145 & IZUMO1 & 19 & $A$ & 0.54 & 0.21 & 0.04 & $1.57 \times 10^{-9}$ \\
\hline Smoking (number) [7] & rs1051730 & CHRNA3 & 15 & $A$ & 0.35 & 1.021 & 0.056 & $2.75 \times 10^{-73}$ \\
\hline Smoking (number) [7] & rs1329650 & LOC100188947 & 10 & G & 0.72 & 0.367 & 0.059 & $5.67 \times 10^{-10}$ \\
\hline Smoking (number) [7] & rs3733829 & CYP2A6 & 19 & G & 0.36 & 0.333 & 0.058 & $1.04 \times 10^{-8}$ \\
\hline Smoking initiation [7] & rs6265 & BDNF & 11 & C & 0.79 & 0.061 & 0.011 & $1.84 \times 10^{-8}$ \\
\hline Smoking cessation [7] & rs3025343 & DBH & 9 & G & 0.84 & 0.121 & 0.022 & $3.56 \times 10^{-8}$ \\
\hline Sleep [8] & rs1191685 & PAX8 & 2 & C & 0.37 & 2.87 & 0.47 & $1.06 \times 10^{-9}$ \\
\hline Sleep [8] & rs2394403 & CBWD & 6 & $C$ & 0.8 & 3.07 & 0.56 & $4.39 \times 10^{-8}$ \\
\hline Sleep [8] & rs4248149 & CBWD & 6 & $\mathrm{~T}$ & 0.8 & 3.08 & 0.56 & $3.95 \times 10^{-8}$ \\
\hline Sleep [8] & rs4587207 & CBWD & 6 & A & 0.8 & 3.14 & 0.56 & $2.02 \times 10^{-8}$ \\
\hline
\end{tabular}

Smoking initiation (ever versus never smokers); Smoking cessation (former versus current smokers) 


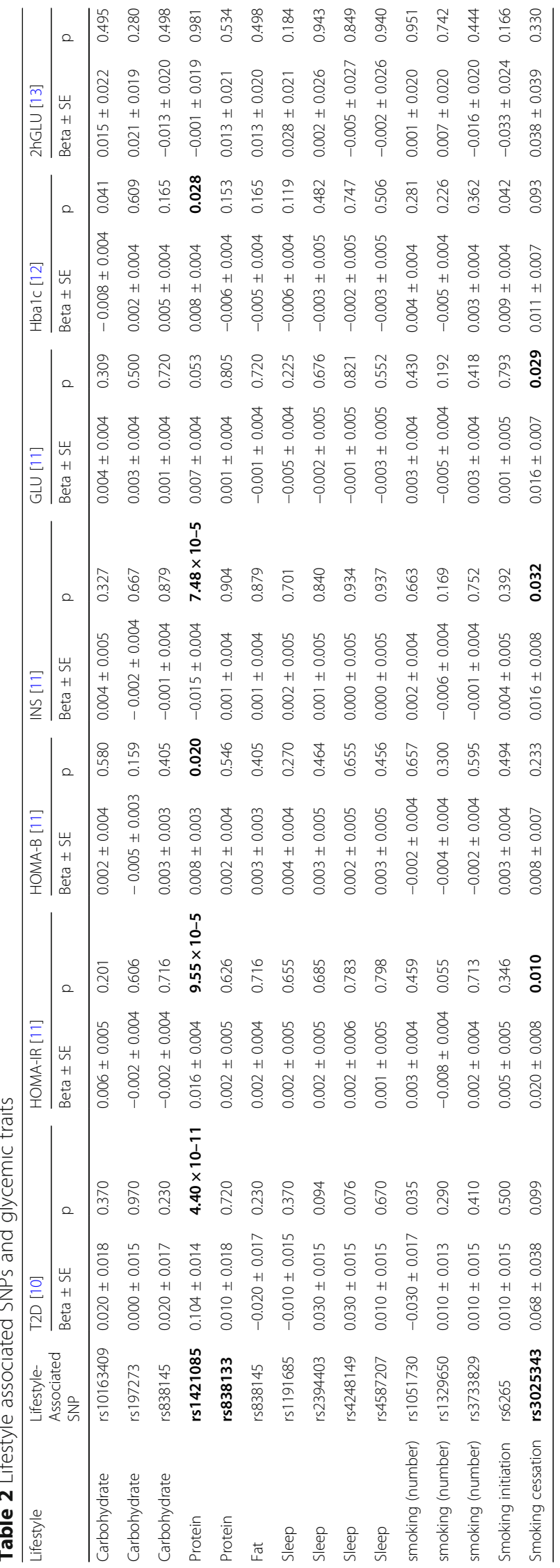




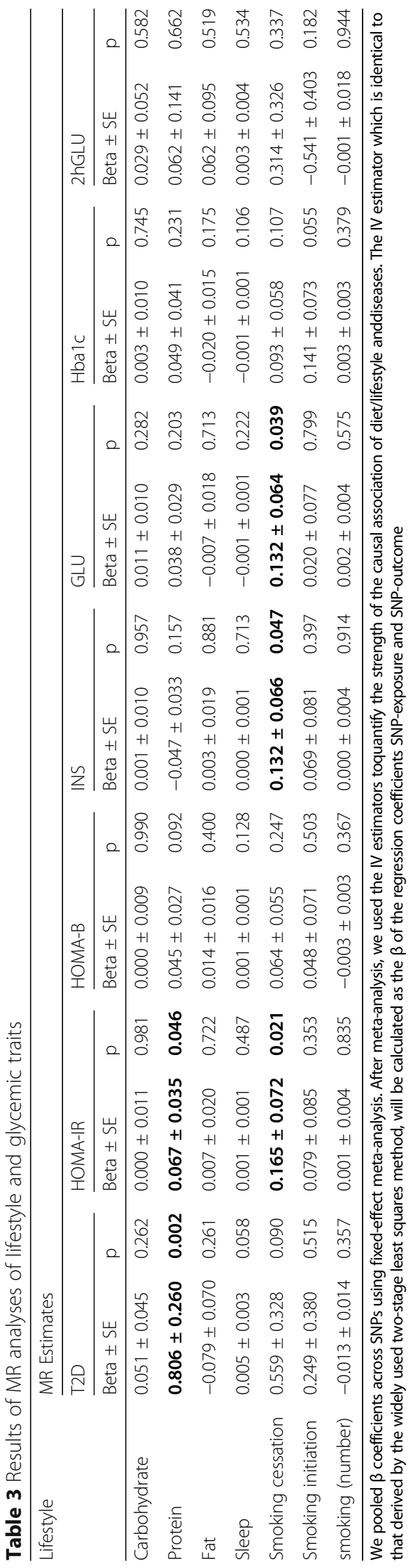


level of insulin resistance. These results provide evidence supporting a causal role for higher protein intake and smoking cession in diabetes, and suggest that individuals at risk for diabetes to keep healthy lifestyle.

Previous research efforts have focused on macronutrient intake in relation to type 2 diabetes risk, [19] but mainly on relative carbohydrate and fat content. Meanwhile, high-protein diets may contribute to disturbance of glucose metabolism. Previous studies addressing dietary protein and diabetes risk focused mainly on highprotein food groups, such as meat and soy. For example, red processed meat intake was related to increased diabetes risk [20-22]. In addition, another study found an increased diabetes risk with higher intake of animal protein and no association with vegetable protein intake, [23] whereas intake of legumes and soy decreased diabetes risk in Asians [24]. However, the Nurses Health Study II did not find such an association, [25] suggesting divergent effects of animal and vegetable protein. The contradictory findings may reflect reverse causation bias and confounding effects. Interestingly, the European Prospective Investigation into Cancer and Nutrition (EPIC)-NL study found that high total protein intake was associated with increased diabetes risk, [22] and suggested that this relation was not explained by specific protein sources such as meat [22]. However, the causality between protein intake and risk of diabetes is not documented. Furthermore, several trials showed beneficial effect of high protein intake on glycemic traits, [26, 27] whereas, high protein diet did not decrease hemoglobin A1c and fasting plasma glucose, and increase insulin sensitivity, [28] making the conclusion difficulty. In the present MR analysis, we found that protein intake was causally associated with increased risk of diabetes and insulin resistance. Our results were generated by using the large scale GWAS summary results, which suggest the robustness of our findings. Our findings for protein intake are generally consistent with those based on prospective observational studies, which tend to report increased risk of diabetes. In addition, reliable GWAS identified genetic variants for protein intake were used as instrumental variables to infer the causality, therefore, our findings were protected from bias such as confounding and reverse causation [4].

Cigarette smoking is an established predictor of incident T2DM. Therefore, smoking cessation should be associated with a decrease in the risk of T2DM. However, smoking cessation is associated with substantial weight gain, [29] which could increase diabetes risk. Several studies have found an increased diabetes risk after smoking cessation [2, 30-32]. However, residual confounding is possible even with meticulous adjustment for established diabetes risk factors in the observational studies. Interestingly, a systematic review and meta-analysis of data from randomised controlled trials of smoking cessation in adults with diabetes found that pooled results did not provide evidence of efficacy for smoking cessation interventions in people with diabetes [33]. Therefore, the causality between smoking cessation and risk of diabetes are still unknown. Using GWAS identified genetic variants for smoking cessation as instrumental variable, our MR analysis provided robust evidence to support that smoking cessation might cause increased risk of T2DM. These findings may carry important public health implications. Smokers at risk for diabetes who quit should receive advice about avoiding weight gain and about diabetes prevention and early detection.

However, our MR did not observe causal association of other lifestyle factors such as fat and carbohydrate intakes and number of smoking cigarettes, smoking initiation, sleep duration, and morning person with risk of T2DM. These findingsare generally contradictory to those based on previous prospective studies, which tend to report increased risk for diabetes in individuals with smoking, both shorter and longer sleep. The contradictory findings may reflect confounding effects, e.g. due to cases being slightly older than controls, or reverse causation bias in the retrospective studies, whereby lifestyle changes arise as a result of disease.

Given the random distribution of genotypes in the general population with respect to lifestyle and other environmental factors, the results of the MR analysis may offer some of the best evidence to assess the causal role of protein and smoking cession in T2DM etiology since the results are less likely to be biased by confounding or reverse causation than traditional observational epidemiological study designs. By employing the twosample MR approach, we were able to increase statistical power by selecting summary statistics from the largest GWAS studies for T2DM (DIAGRAM, $n=149,821$ ) and glycemic traits (MAGIC, $n=133,010$ ).

Our study is subject to some limitations. First, our results assume that the samples used to define the instrumental variable for diet/lifestyle and the samples from consortium used to estimate the genetic association with disease/traits are from the same population. Second, our results mightbeconfoundedby pleiotropic pathways. ${ }^{35} \mathrm{We}$ cannot entirely rule out this possibility. Third, our study assumed a linear relationship between diet/lifestyle and T2DM and glycemic traits. In this study, we could not investigate nonlinear effects of diet/lifestyle. Fourth, we cannot exclude the possibility of sample overlap since both DIAGRAM and the MAGIC consortiums used samples from the lifestyle GWASstudy. Therefore, this might introduce bias into our results. Finally, we cannot rule out chance of violation of any of the three MR assumptions, which would potentially bias the magnitude of the estimated causal association [34]. 


\section{Conclusions}

In summary, these results provide evidence supporting a causal role for higher protein intake and smoking cession in diabetes. This provides further rationale for individuals at risk for diabetes to keep healthy lifestyle. However, whether different sources of protein diet, or duration of smoking cession, is mediating these relationships warrants further investigations.

\section{Acknowledgements}

Not applicable.

\section{Funding}

This study was supported by the National University of Singapore start-up grant: R-608-000-139-133 and Singapore Ministry of Education Tier 1 grant: R-608-000$161-114$

\section{Availability of data and materials}

The datasets used and/or analysed during the current study available from the corresponding author on reasonable request.

\section{Authors' contributions}

$\mathrm{RD}$ and $\mathrm{TH}$ designed the study, drafted the study protocol, and planned analyses. $\mathrm{RD}$ and $\mathrm{TH}$ wrote the first draft of the paper. RD and $\mathrm{TH}$ conducted the data collection and combined statistical analysis. RD and $\mathrm{TH}$ contributed to study design, preparation of manuscript. All of the authors contributed to the design, analysis, interpretation, and drafting of this paper. All authors had reviewed and approved the drafts of the paper.

\section{Ethics approval and consent to participate}

Not applicable.

\section{Consent for publication}

Not applicable.

\section{Competing interests}

The authors declare that they have no competing interests.

\section{Publisher's Note}

Springer Nature remains neutral with regard to jurisdictional claims in published maps and institutional affiliations.

\section{Author details}

${ }^{1}$ Department of Intensive Care Medicine, The First Hospital of China Medical University, Liaoning 110001, China. ${ }^{2}$ Department of Epidemiology and Biostatistics, School of Public Health, Peking University, Beijing 100191, China. ${ }^{3}$ Department of Otolaryngology, The First Hospital of China Medical University, Liaoning 110001, China.

Received: 12 June 2017 Accepted: 22 January 2018

Published online: 29 January 2018

\section{References}

1. Hu FB, Manson JE, Stampfer MJ, Colditz G, Liu S, Solomon CG, Willett WC. Diet, lifestyle, and the risk of type 2 diabetes mellitus in women. $\mathrm{N}$ Engl J Med. 2001;345:790-7.

2. Yeh HC, Duncan BB, Schmidt MI, Wang NY, Brancati FL. Smoking, smoking cessation, and risk for type 2 diabetes mellitus: a cohort study. Ann Intern Med. 2010;152:10-7.

3. Shan Z, Ma H, Xie M, Yan P, Guo Y, Bao W, Rong Y, Jackson CL, Hu FB, Liu L, Sleep duration and risk of type 2 diabetes: a meta-analysis of prospective studies. Diabetes Care. 2015;38:529-37.

4. Lawlor DA, Harbord RM, Sterne JA, Timpson N, Davey Smith G. Mendelian randomization: using genes as instruments for making causal inferences in epidemiology. Stat Med. 2008:27:1133-63.

5. Chu AY, Workalemahu T, Paynter NP, Rose LM, Giulianini F, Tanaka T, Ngwa JS, Qi Q, Curhan GC, Rimm EB, et al. Novel locus including FGF21 is associated with dietary macronutrient intake. Hum Mol Genet. 2013;22:1895-902.
6. Tanaka T, Ngwa JS, van Rooij FJ, Zillikens MC, Wojczynski MK, Frazier-wood AC, Houston DK, Kanoni S, Lemaitre RN, Luan J, et al. Genome-wide metaanalysis of observational studies shows common genetic variants associated with macronutrient intake. Am J Clin Nutr. 2013:97:1395-402.

7. Tobacco and Genetics Consortium. Genome-wide meta-analyses identify multiple loci associated with smoking behavior. Nat Genet. 2010:42:441-7.

8. Gottlieb DJ, Hek K, Chen TH, Watson NF, Eiriksdottir G, Byrne EM, Cornelis M, Warby SC, Bandinelli S, Cherkas L, et al. Novel loci associated with usual sleep duration: the CHARGE consortium genome-wide association study. Mol Psychiatry. 2015;20:1232-9.

9. Welter D, MacArthur J, Morales J, Burdett T, Hall P, Junkins H, Klemm A, Flicek P, Manolio T, Hindorff L, Parkinson H. The NHGRI GWAS catalog, a curated resource of SNP-trait associations. Nucleic Acids Res. 2014:42:D1001-6.

10. Mahajan A, Go MJ, Zhang W, Below JE, Gaulton KJ, Ferreira T, Horikoshi M, Johnson AD, Ng MC, Prokopenko I, et al. Genome-wide trans-ancestry metaanalysis provides insight into the genetic architecture of type 2 diabetes susceptibility. Nat Genet. 2014;46:234-44.

11. Dupuis J, Langenberg C, Prokopenko I, Saxena R, Soranzo N, Jackson AU, Wheeler E, Glazer NL, Bouatia-Naji N, Gloyn AL, et al. New genetic loci implicated in fasting glucose homeostasis and their impact on type 2 diabetes risk. Nat Genet. 2010;42:105-16.

12. Soranzo N, Sanna S, Wheeler E, Gieger C, Radke D, Dupuis J, Bouatia-Naji N,

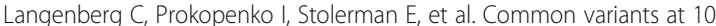
genomic loci influence hemoglobin a(1)(C) levels via glycemic and nonglycemic pathways. Diabetes. 2010;59:3229-39.

13. Saxena R, Hivert MF, Langenberg C, Tanaka T, Pankow JS, Vollenweider P, Lyssenko V, Bouatia-Naji N, Dupuis J, Jackson AU, et al. Genetic variation in GIPR influences the glucose and insulin responses to an oral glucose challenge. Nat Genet. 2010;42:142-8.

14. Hartwig FP, Davies NM, Hemani G, Davey Smith G. Two-sample Mendelian randomization: avoiding the downsides of a powerful, widely applicable but potentially fallible technique. Int J Epidemiol. 2016;45:1717-26.

15. Davey Smith G, Hemani G. Mendelian randomization: genetic anchors for causal inference in epidemiological studies. Hum Mol Genet. 2014;23:R89-98.

16. Mokry LE, Ross S, Ahmad OS, Forgetta V, Smith GD, Goltzman D, Leong A, Greenwood CM, Thanassoulis G, Richards JB. Vitamin D and risk of multiple sclerosis: a Mendelian randomization study. PLoS Med. 2015;12:e1001866.

17. Burgess S, Butterworth A, Thompson SG. Mendelian randomization analysis with multiple genetic variants using summarized data. Genet Epidemiol. 2013;37:658-65

18. Patsopoulos NA, Evangelou E, loannidis JP. Sensitivity of between-study heterogeneity in meta-analysis: proposed metrics and empirical evaluation. Int J Epidemiol. 2008;37:1148-57.

19. van Dam RM, Willett WC, Rimm EB, Stampfer MJ, Hu FB. Dietary fat and meat intake in relation to risk of type 2 diabetes in men. Diabetes Care. 2002;25:417-24

20. Fung TT, Schulze M, Manson JE, Willett WC, Hu FB. Dietary patterns, meat intake, and the risk of type 2 diabetes in women. Arch Intern Med. 2004;164:2235-40.

21. Micha R, Michas G, Mozaffarian D. Unprocessed red and processed meats and risk of coronary artery disease and type 2 diabetes-an updated review of the evidence. Curr Atheroscler Rep. 2012;14:515-24.

22. Sluijs I, Beulens JW, van der AD, Spijkerman AM, Grobbee DE, van der Schouw YT. Dietary intake of total, animal, and vegetable protein and risk of type 2 diabetes in the European prospective investigation into cancer and nutrition (EPIC)-NL study. Diabetes Care. 2010;33:43-8.

23. Song $Y$, Manson JE, Buring JE, Liu S. A prospective study of red meat consumption and type 2 diabetes in middle-aged and elderly women: the women's health study. Diabetes Care. 2004;27:2108-15.

24. Villegas R, Gao YT, Yang G, Li HL, Elasy TA, Zheng W, Shu XO. Legume and soy food intake and the incidence of type 2 diabetes in the shanghai Women's health study. Am J Clin Nutr. 2008:87:162-7.

25. Schulze MB, Liu S, Rimm EB, Manson JE, Willett WC, Hu FB. Glycemic index, glycemic load, and dietary fiber intake and incidence of type 2 diabetes in younger and middle-aged women. Am J Clin Nutr. 2004;80:348-56.

26. Larsen RN, Mann NJ, Maclean E, Shaw JE. The effect of high-protein, lowcarbohydrate diets in the treatment of type 2 diabetes: a 12 month randomised controlled trial. Diabetologia. 2011;54:731-40.

27. Farnsworth E, Luscombe ND, Noakes M, Wittert G, Argyiou E, Clifton PM Effect of a high-protein, energy-restricted diet on body composition, glycemic control, and lipid concentrations in overweight and obese hyperinsulinemic men and women. Am J Clin Nutr. 2003;78:31-9. 
28. Sargrad KR, Homko C, Mozzoli M, Boden G. Effect of high protein vs high carbohydrate intake on insulin sensitivity, body weight, hemoglobin A1c, and blood pressure in patients with type 2 diabetes mellitus. J Am Diet Assoc. 2005;105:573-80.

29. O'Hara P, Connett JE, Lee WW, Nides M, Murray R, Wise R. Early and late weight gain following smoking cessation in the lung health study. Am J Epidemiol. 1998;148:821-30.

30. Pan A, Wang Y, Talaei M, Hu FB, Wu T. Relation of active, passive, and quitting smoking with incident type 2 diabetes: a systematic review and meta-analysis. Lancet Diabetes Endocrinol. 2015;3:958-67.

31. Stein JH, Asthana A, Smith SS, Piper ME, Loh WY, Fiore MC, Baker TB. Smoking cessation and the risk of diabetes mellitus and impaired fasting glucose: threeyear outcomes after a quit attempt. PLoS One. 2014;9:e98278.

32. Sung $Y T$, Hsiao CT, Chang IJ, Lin YC, Yueh CY. Smoking cessation carries a short-term rising risk for newly diagnosed diabetes mellitus independently of weight gain: a 6-year retrospective cohort study. J Diabetes Res. 2016; 2016:3961756.

33. Nagrebetsky A, Brettell R, Roberts N, Farmer A. Smoking cessation in adults with diabetes: a systematic review and meta-analysis of data from randomised controlled trials. BMJ Open. 2014;4:e004107.

34. Burgess S, Butterworth AS, Thompson JR. Beyond Mendelian randomization: how to interpret evidence of shared genetic predictors. J Clin Epidemiol. 2016;69:208-16.

\section{Submit your next manuscript to BioMed Central and we will help you at every step:}

- We accept pre-submission inquiries

- Our selector tool helps you to find the most relevant journal

- We provide round the clock customer support

- Convenient online submission

- Thorough peer review

- Inclusion in PubMed and all major indexing services

- Maximum visibility for your research

Submit your manuscript at www.biomedcentral.com/submit

) Biomed Central 